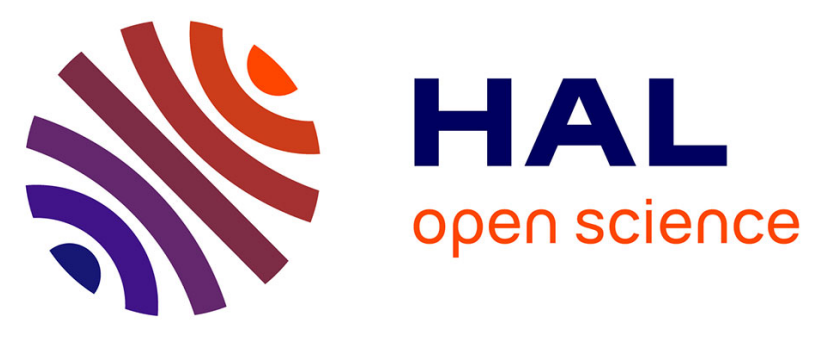

\title{
Elucidating 1S1R operation to reduce the read voltage margin variability by stack and programming conditions optimization
}

J Minguet Lopez, L. Hudeley, L. Grenouillet, D Alfaro Robayo, J. Sandrini, G. Navarro, M. Bernard, C. Carabasse, D. Deleruyelle, N. Castellani, et al.

\section{To cite this version:}

J Minguet Lopez, L. Hudeley, L. Grenouillet, D Alfaro Robayo, J. Sandrini, et al.. Elucidating $1 \mathrm{~S} 1 \mathrm{R}$ operation to reduce the read voltage margin variability by stack and programming conditions optimization. 2021 IEEE International Reliability Physics Symposium (IRPS), Mar 2021, Monterey, United States. 10.1109/IRPS46558.2021.9405195 . hal-03333678

\section{HAL Id: hal-03333678 https://hal.science/hal-03333678}

Submitted on 3 Sep 2021

HAL is a multi-disciplinary open access archive for the deposit and dissemination of scientific research documents, whether they are published or not. The documents may come from teaching and research institutions in France or abroad, or from public or private research centers.
L'archive ouverte pluridisciplinaire HAL, est destinée au dépôt et à la diffusion de documents scientifiques de niveau recherche, publiés ou non, émanant des établissements d'enseignement et de recherche français ou étrangers, des laboratoires publics ou privés. 


\title{
Elucidating 1S1R operation to reduce the read voltage margin variability by stack and programming conditions optimization
}

\author{
J. Minguet Lopez, L. Hudeley, L. Grenouillet, D. Alfaro Robayo, J. Sandrini, G. Navarro, M. Bernard, C. Carabasse, D. \\ Deleruyelle $^{3}$, N. Castellani, M. Bocquet ${ }^{2}$, J. M. Portal ${ }^{2}$, E. Nowak, G. Molas \\ CEA, LETI, MINATEC Campus, GRENOBLE, France, joel.minguetlopez@ cea.fr, gabriel.molas@ cea.fr \\ ${ }^{2}$ Aix Marseille Univ, Université de Toulon, CNRS, IM2NP, Marseille, France \\ ${ }^{3}$ INL CNRS, INSA Lyon, France
}

\begin{abstract}
S1R operation behavior and read margin variability are elucidated for the first time to our knowledge. To this aim, an extensive experimental study is performed on $\mathrm{HfO}_{2}$ OxRAM technology co-integrated with GSSN-based Ovonic Threshold Selector (OTS) in 4kb memory arrays, supported by deep theoretical analysis. A semi-analytical dynamic model is developed for selector operation, describing OTS switching variability. The voltage repartition in the 1S1R stack during the switching operation is clarified. The 1S1R read voltage margin is quantified statistically, based on OTS switching voltage dispersion, OxRAM variability and margin degradation during endurance. Based on this theoretical and experimental study, 1S1R figures of merit (overall functionality, reliability, and maximum bank size) are optimized based on OTS and OxRAM devices' features and programming conditions.
\end{abstract}

Index Terms--chalcogenide, crossbar, OTS, operation variability, resistive RAM

\section{INTRODUCTION}

Resistive Random-Access Memory (RRAM) is one of the most promising candidates for high-density crossbar architectures [1-2] targeting memory and neuromorphic circuit applications [3]. In this aim, OxRAM co-integration with Ovonic Threshold Switching (OTS) back-end selector is one of the most competitive approaches for the sneak-path current reduction in a high-density crossbar array. In previous works, OTS (1S) was successfully co-integrated with $\mathrm{HfO}_{2}$ based OxRAM (1R) [4-5], demonstrating promising functionality for large crossbar arrays. However, among the various device parameters, read voltage margin was identified as one of the most critical feature for 1S1R stack. Beyond device functionality, it is thus mandatory to deeply analyze and understand how both OxRAM and OTS affect the reading window. In this work, we present a theoretical and experimental statistical study of the 1S1R read voltage margin and propose to reduce its variability through device lifetime, by optimizing the stack and programming conditions. Moreover, knowing optimized stack and programming conditions, we provide general guidelines to guarantee the overall functionality and reliability of crossbar arrays depending on their size.

\section{TECHNOLOGICAL DETAILS}

OxRAM is co-integrated with OTS back-end selector in $4 \mathrm{~kb} 1 \mathrm{~S} 1 \mathrm{R}$ matrix configuration. A transistor is used as current limiter. Magnetron sputtered Ge-Se-Sb-N (GSSN) alloy is used as OTS, sandwiched between two Carbon electrodes to improve endurance [6]. 5nm-thick $\mathrm{ALD} \mathrm{HfO}_{2}$ is integrated with a 5nm PVD Ti scavenging layer for the OxRAM device [7]. Showed in Fig.1 (a), (b) and (c), SEM top view, TEM cross section and EDX image of the stack demonstrate the consistent co-integration of all deposited layers.

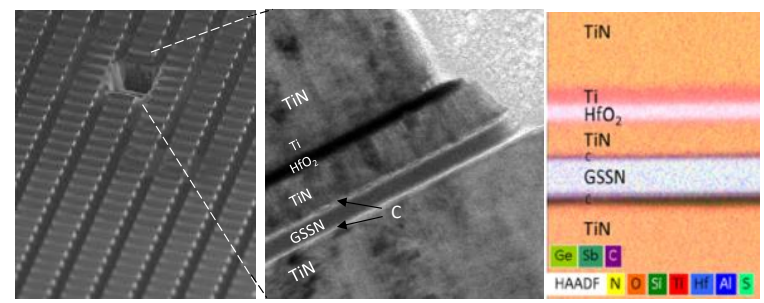

Fig. 1. (a) SEM top view of $4 \mathrm{~kb} 1 \mathrm{~S} 1 \mathrm{R}$ array composed of $\mathrm{HfO}_{2}$ based OxRAM and GeSeSbN (GSSN) based OTS. (b) TEM cross section of a 1S1R stack. (c) EDX image of the 1S1R stack showing the elements constituting the layers.

\section{RESULTS AND DISCUSSION}

A. OTS and OXRAM switching characteristics

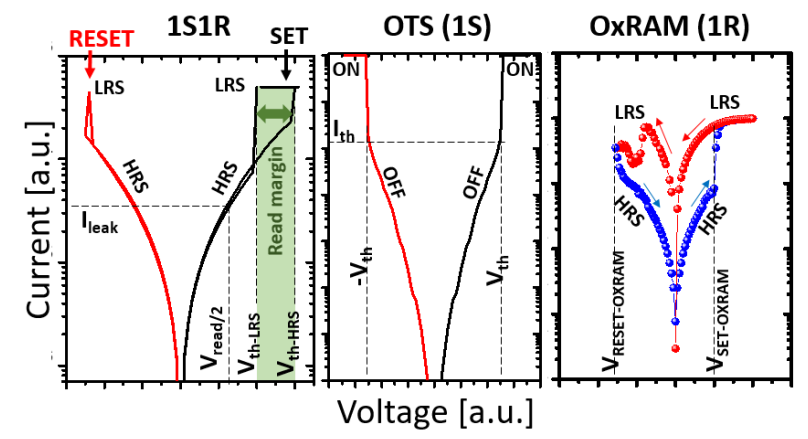

Fig. 2. IV of 1S1R, 1S (GSSN OTS) and 1R (OxRAM, blue for HRS, red for LRS) devices showing the device's key electrical parameters used in this work.

Fig.2 shows typical IV switching characteristics of our $1 \mathrm{~S} 1 \mathrm{R}, 1 \mathrm{R}$ and $1 \mathrm{~S}$ devices together with the key electrical parameters used in this work. In particular, the reading voltage should be comprised between $\mathrm{V}_{\text {th-LRS }}$ and $\mathrm{V}_{\text {th-HRS }}$ (respectively the $1 \mathrm{~S} 1 \mathrm{R}$ switching voltages in low and high resistive states) defining the read margin. 


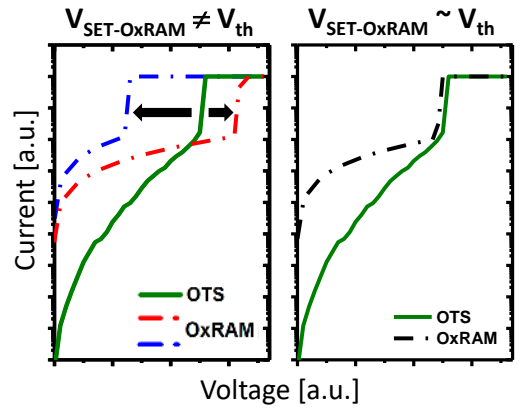

Fig. 3. IV SET switching characteristics of $1 \mathrm{~S}$ and $1 \mathrm{R}$ devices. While preserving satisfactory device insulating properties, switching voltages for memory and selector devices should be of the same order to avoid voltage stress in the stack.

Fig.3 plots the IV SET characteristics of $1 \mathrm{R}$ and $1 \mathrm{~S}$ devices. The OTS switching voltage $\mathrm{V}_{\text {th }}$ being linked to its isolating properties [6], 1S1R optimization strategies must engineer both OTS and OxRAM switching voltages without compromising OTS insulating properties.

\section{B. Semi-analytical model for OTS switching}

Semi-analytical simulations of $1 \mathrm{~S}$ devices' operations are developed based on filamentary field-driven switching theory $[8,4]$. OTS switching is described by the successive nucleation of metastable domains within the GSSN chalcogenide layer. OFF-to-ON switching (resp. ON-to-OFF relaxation) through filament nucleation (resp. dissolution), together with the main equations implemented in the model, are detailed in Fig.4 and Fig.5. After firing, once the OTS returns to the OFF state, a residual filament remains.

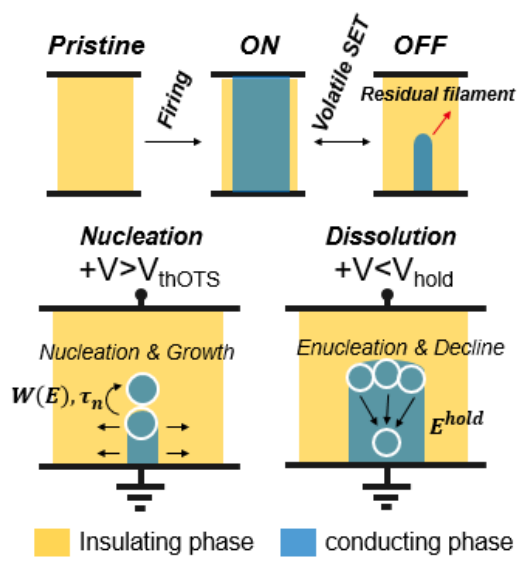

Fig. 4. Description of the semi-analytical model used to simulate the switching dynamics of the OTS: schematics of the simulated OTS material in pristine, ON and OFF states. The conductive state being insured by the growth of a conductive filament, filament dynamics for device switch to ON state (nucleation) and relaxation to OFF state (dissolution) are presented.

$$
\begin{aligned}
& \text { Height nucleation barrier } \\
& \text { (free energy maximum) } \\
& W(E)=\frac{W_{0 \_ \text {height }}}{\left(1+\frac{H^{3}}{8 * W_{0 \_ \text {height }}} \epsilon_{r} E^{2}\right)^{2}} \\
& \text { Radius nucleation barrier }
\end{aligned}
$$

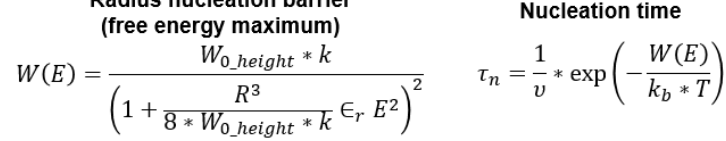

$$
\begin{aligned}
& E=\text { electric field }[\mathrm{V} / \mathrm{m}] \\
& W_{0 \text { height }}=E_{0}^{\text {hold }}=\text { zero field nucleation barrier }=2.75 \mathrm{eV} \\
& k=\text { depolarizing factor influence }=1.1 \\
& H=\text { domain critical height }=6 \mathrm{~nm} \\
& R=\text { domain critical radius }=3 \mathrm{~nm} \\
& \epsilon_{r}=\text { relative permittivity }=10 \quad \sigma_{V_{\text {hold }}}=0.9 \mathrm{~V}
\end{aligned}
$$

Fig.5. Description of the semi-analytical model used to simulate the switching dynamics of the OTS: equations implemented in the model.

Fig.6 shows experimental and simulated OTS firing and threshold voltage evolution with GSSN thickness. A residual filament of $\sim 44 \%$ of the GSSN thickness after firing process is used for OTS threshold $\mathrm{V}_{\text {th }}$ simulation, satisfactorily capturing experimental results. Assuming a standard deviation of $0.15 \mathrm{eV}$ for holding activation energy $\left(E^{\text {hold }}\right), 10 \mathrm{~nm}$-thick GSSN-based experimental and simulated OTS holding voltage $\left(\mathrm{V}_{\text {hold }}\right)$ distributions are presented in Fig.7 (a) with a median of $0.9 \mathrm{~V}$. $\mathrm{V}_{\text {hold }}$ is a key parameter quantifying the voltage that drops on the selector in the ON state. Moreover, both experimental and simulated OTS switching voltage $\mathrm{V}_{\text {th }}$ evolution with voltage sweep rate are presented in Fig.7 (b). All in all, successful agreement between experimental and simulated data is evidenced for pristine, ON and OFF cell resistive states (Fig.4) in quasi-static and dynamic regimes.

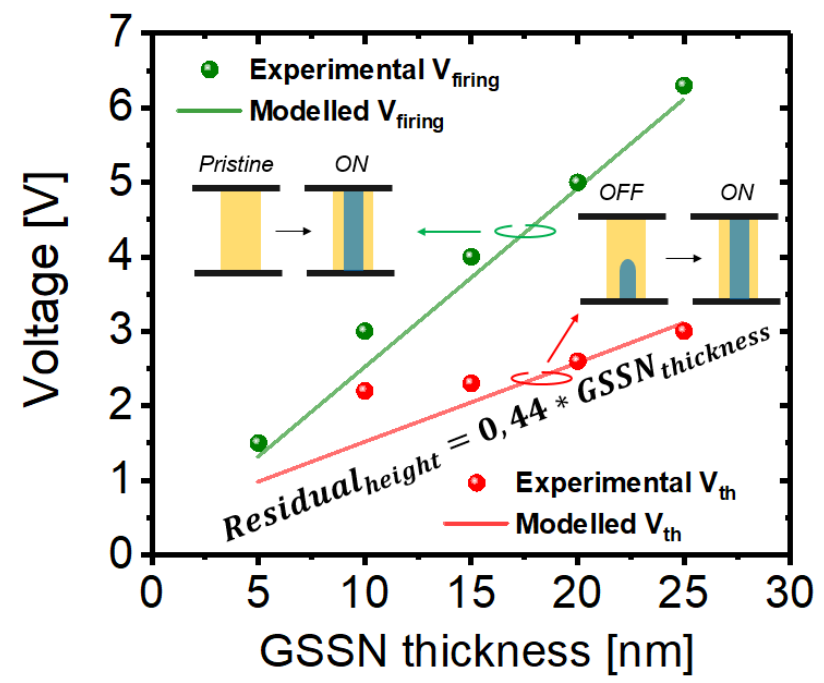

Fig. 6. Experimental and simulated selector firing and threshold voltage as function of GSSN thickness (considering a residual filament of $\sim 44 \%$ of the GSSN thickness after relaxation to OFF state). 

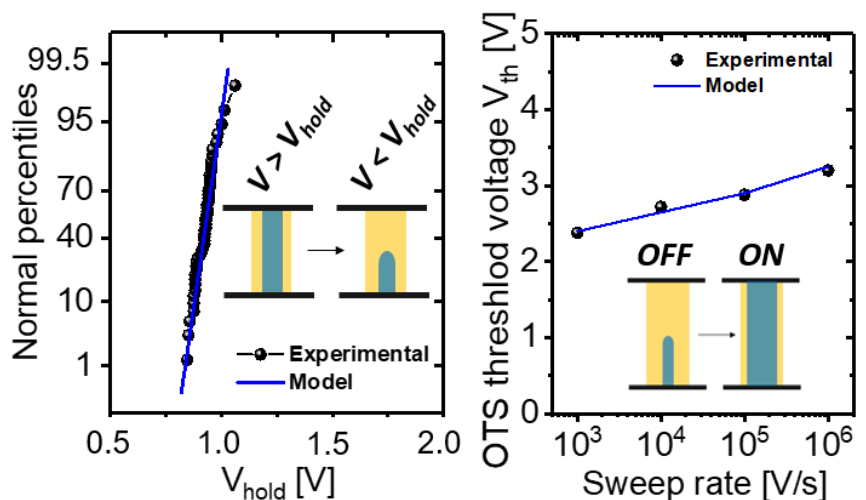

Fig. 7. (a) Experimental and simulated distribution of the OTS holding voltage for $10 \mathrm{~nm}$ GSSN. $0.15 \mathrm{eV}$ of holding activation energy $\left(E^{\text {hold }}\right)$ dispersion is considered for the simulation. (b) Experimental and simulated OTS switching voltage $\left(\mathrm{V}_{\mathrm{th}}\right)$ as function of voltage sweep rate. Faster ramps lead to higher switching voltages.

\section{OXRAM+OTS read voltage margin variability reduction \\ a. ISIR programming conditions impact on read voltage margin}

Fig.8 shows experimental and simulated $\mathrm{V}_{\text {firing, }}, \mathrm{V}_{\text {th-HRS }}$ (high resistive state) and $\mathrm{V}_{\text {th-LRS }}$ (low resistive state) 1S1R switching voltages. $\mathrm{V}_{\text {th-HRS }}>\mathrm{V}_{\text {th-LRS }}$ when the OxRAM is in HRS due to the additional voltage that drops on the OxRAM. The higher $\mathrm{R}_{\mathrm{HRS}}$, the higher $\mathrm{V}_{\text {th-HRS }}$ switching values and so the larger the $1 \mathrm{~S} 1 \mathrm{R}$ read voltage margin $\left(\mathrm{V}_{\text {th-HRS }}-\mathrm{V}_{\text {th-LRS }}\right)$. Particularly, the $1 \mathrm{~S} 1 \mathrm{R}$ reading voltage is comprised within the read voltage margin. $V_{\text {th-HRS }}$ distributions, using OTS modelling (previous section) and OxRAM (Poole Frenkel mechanism) conduction characteristics, are calculated for various $\mathrm{R}_{\mathrm{HRS}}$ (blue discontinuous lines in Fig.8).

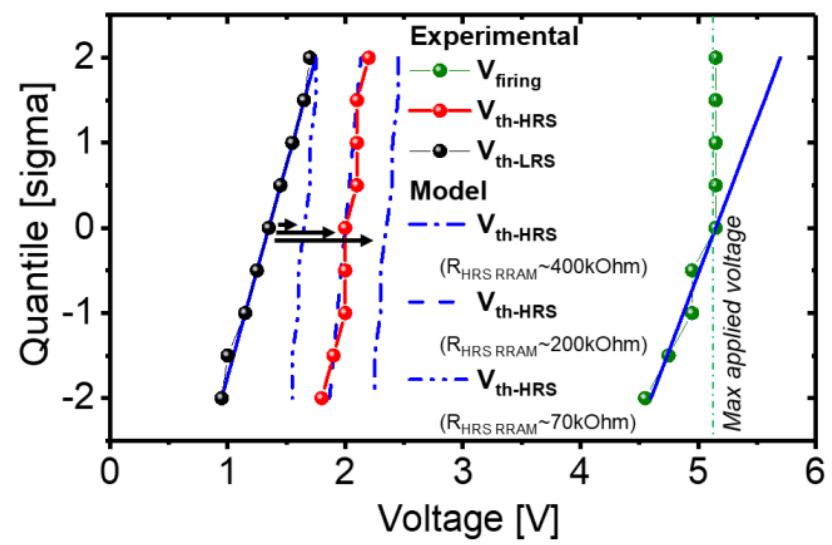

Fig. 8. Distribution of experimental and simulated 1S1R threshold voltages for $10 \mathrm{~nm}$ GSSN and $10 \mathrm{~nm} \mathrm{HfO} \mathrm{H}_{2} . \mathrm{V}_{\text {th-LRS }}$ (resp. $\mathrm{V}_{\text {th-HRs }}$ ) is measured for the OxRAM in the LRS (resp. HRS). For $\mathrm{V}_{\text {firing }}>5.2 \mathrm{~V}$, longer pulse time at fixed voltage is applied to form the $1 \mathrm{~S} 1 \mathrm{R}$. Calculated $\mathrm{V}_{\text {th-HRS }}$ distributions take into account OxRAM R $\mathrm{R}_{\text {HRS }}$.

Moreover, OxRAM $\mathrm{R}_{\mathrm{HRS}}$ variability leads to read voltage margin reduction due to the resulting $\mathrm{V}_{\text {th-HRS }}$ switching voltage dispersion (Fig.8), which requires SET and RESET optimization to maximize $\mathrm{V}_{\text {th-HRS }}$ and reduce its dispersion over lifetime.

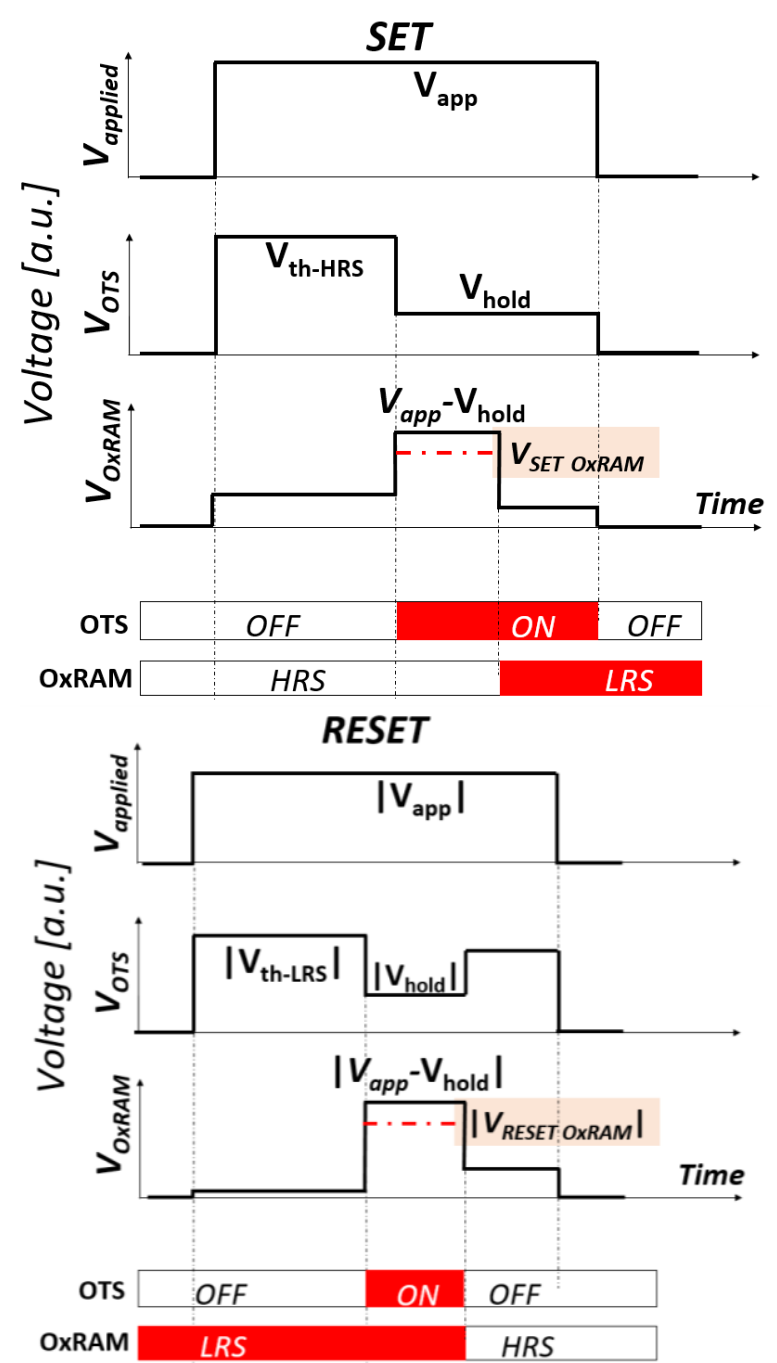

Fig.9. Voltage repartition in $1 \mathrm{~S} 1 \mathrm{R}$ structures during programming and respective states of OTS and OxRAM during SET and RESET

Once the OTS becomes conductive, $\mathrm{V}_{\text {hold }}$ drops on the OTS and $\mathrm{V}_{\text {app }}-\mathrm{V}_{\text {hold }}$ on the OxRAM (Fig.9). It is thus important to control the stack features and programming voltages, so that $\mathrm{V}_{\text {app }}>\mathrm{V}_{\text {th-HRS }}$ and $\mathrm{V}_{\text {app }}>\mathrm{V}_{\text {th-LRS }}$ to efficiently open the OTS, while $\mathrm{V}_{\text {app }}-\mathrm{V}_{\text {hold }}$ (voltage that drops on the OxRAM) has to be compatible with a functional operation of the OxRAM: sufficient for a proper programming operation but not too high to prevent device early degradation. Fig.10 (a) shows how $\mathrm{V}_{\text {RESET }}$ seen by the OxRAM (which is a function of $\mathrm{V}_{\text {app }}$ ) affects 1S1R window margin (see Fig.10 (b)) and reading yield (probability that $\mathrm{V}_{\text {th-HRS }}>\mathrm{V}_{\text {th-LRS }}$ on the array) at $10^{4}$ operating cycles. In particular, a minimum voltage seen by OxRAM ensures a $1 \mathrm{~S} 1 \mathrm{R}$ window margin of $\sim 1 \mathrm{~V}$ while a too high voltage seen by OxRAM device is detrimental for the reading yield. 

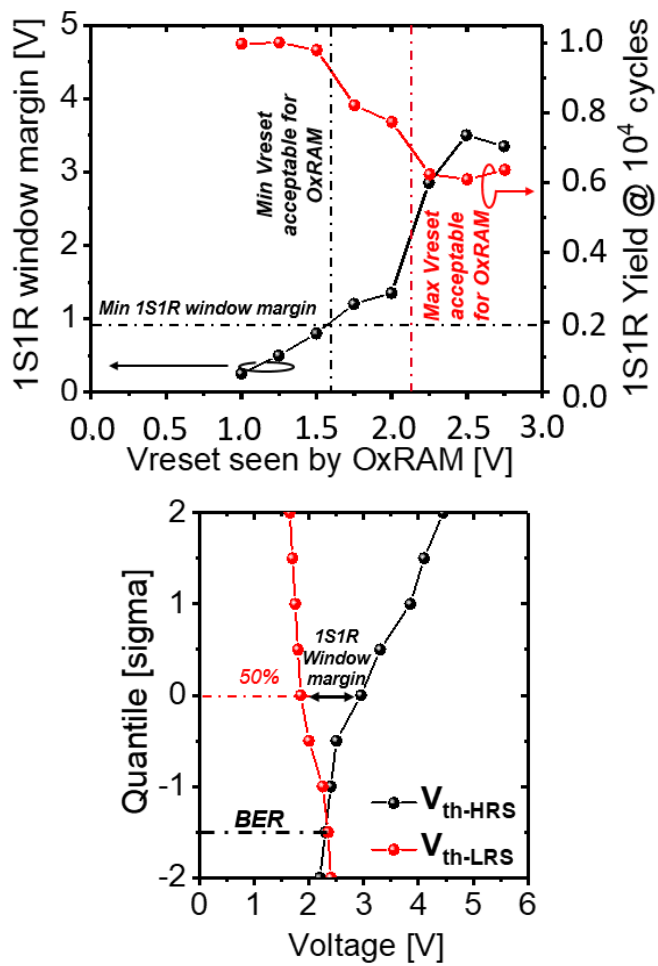

Fig. 10. (a) Calculated $1 \mathrm{~S} 1 \mathrm{R}$ window margin and reading yield at $10^{4}$ operating cycles' evolution with voltage seen by OxRAM for RESET operation. Reading yield is defined as the probability for $\mathrm{V}_{\text {th-HRS }}$ and $\mathrm{V}_{\text {th-LRS }}$ to be distinct so that the resistive state of the device can be read. (b) Illustrative $\mathrm{V}_{\text {th-HRS }}$ and $\mathrm{V}_{\text {th-LRS }} 1 \mathrm{~S} 1 \mathrm{R}$ distributions (with intentional poor performances), detailing window margin and BER (=1-yield).

Fig.11 concretizes the tradeoff between 1S1R read margin (calculated from $1 \mathrm{R}$ window margin) and maximum measured endurance (with stable window). Up to $4 \mathrm{~V} 1 \mathrm{~S} 1 \mathrm{R}$ read voltage margin values can be reached if only $10^{3}$ cycles are needed, and up to $10^{7}$ cycles can be achieved if $\sim 500 \mathrm{mV}$ read margin is tolerated.

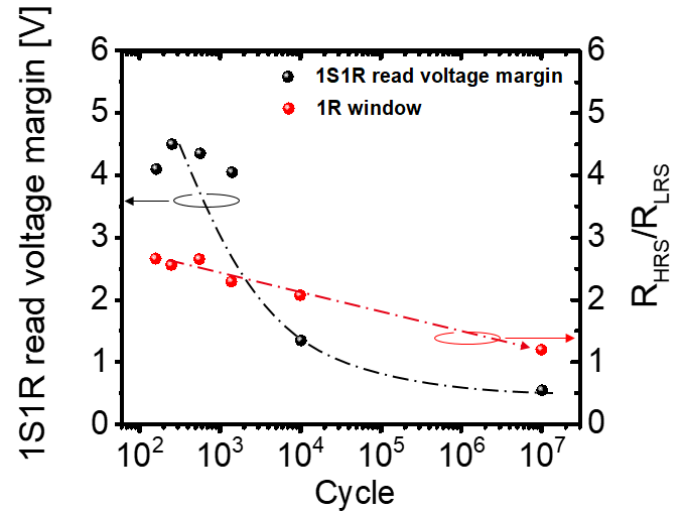

Fig. 11. Evolution of experimental OxRAM window margin (red symbols) as function of maximum number of cycles insuring stable window, and corresponding calculated 1S1R mean read voltage margin (black symbols).

\section{b. $\quad I S$ and $1 R$ stack influence on read voltage margin}

Based on OxRAM endurance, $\mathrm{V}_{\text {th-HRS }}$ and $\mathrm{V}_{\text {th-LRS }}$ distributions are calculated from OxRAM $R_{H R S}$ and $R_{L R S}$ statistics for a wide range of $V_{S E T}$ and $V_{\text {RESET }}$ conditions, allowing to extract $1 \mathrm{~S} 1 \mathrm{R}$ read margin and BER heatmaps (Fig.12). While strong $\mathrm{V}_{\text {RESET }}$ enlarges 1S1R read window margin, poor endurance with high BER is obtained due to OxRAM early degradation. White dots indicate the voltages the OxRAM undergoes after OTS switching during 1S1R operation for various GSSN thicknesses. Therefore, $10 \mathrm{~nm}$ and $15 \mathrm{~nm}$-thick GSSN co-integration with $5 \mathrm{~nm}$-thick $\mathrm{HfO}_{2}$ ensures reliable read window margin features with no early degradation. Indeed, for $>15 \mathrm{~nm}$ GSSN, BER dramatically increases due to the strong voltage required to open the OTS that degrades the OxRAM after cycling.
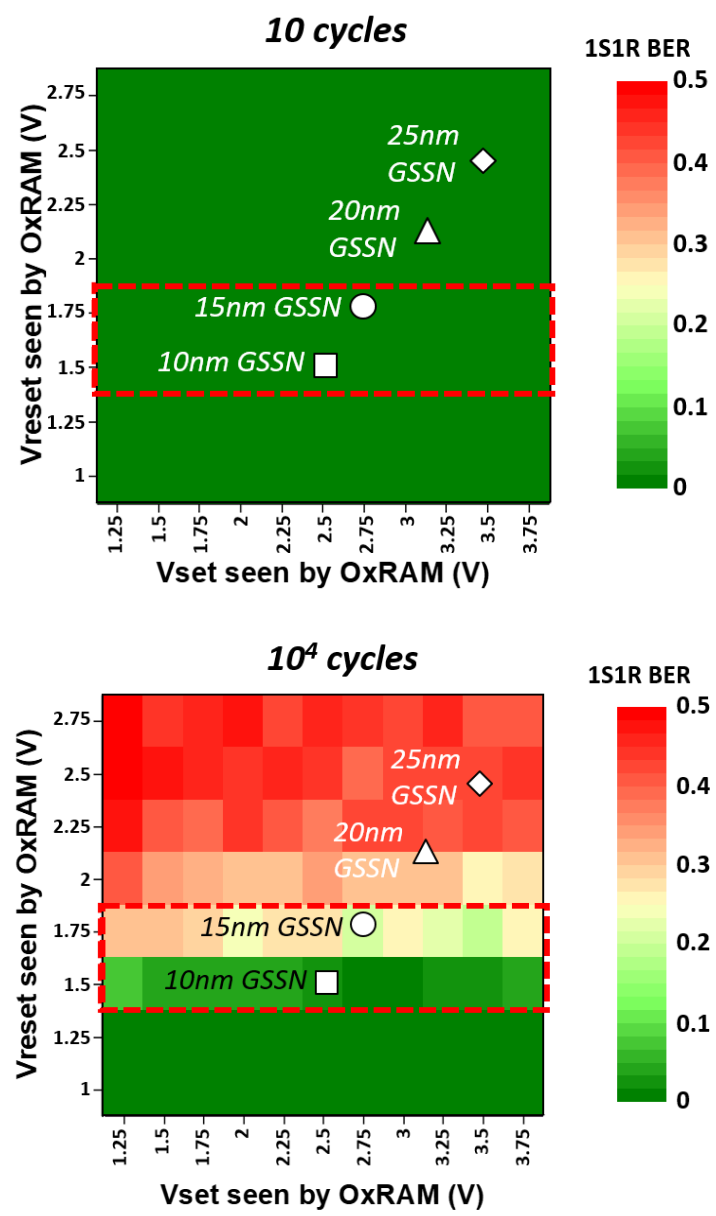

Fig. 12. 1S1R BER evaluation based on SET and RESET programming strategies through cycling (for 100ns pulses). Aggressive RESET strategies lead to excessive voltage drop to OxRAM devices, inducing 1S1R BER early degradation. Fail occurs when $\mathrm{V}_{\text {th-HRS }}<\mathrm{V}_{\text {th-LRs }}$. Symbols illustrate the voltages the OxRAM undergoes after GSSN switches to the ON state, for various selector thicknesses. 


\section{c. ISIR figures of merit on read voltage margin}

Based on these analyses, 1S1R figures of merit are proposed. Fig.13 illustrates the functional SET and RESET operating regions, both to open the OTS and program the OxRAM without degradation (fig.12), allowing to adjust GSSN thickness. 10-15nm GSSN appears here to be the functional working range.
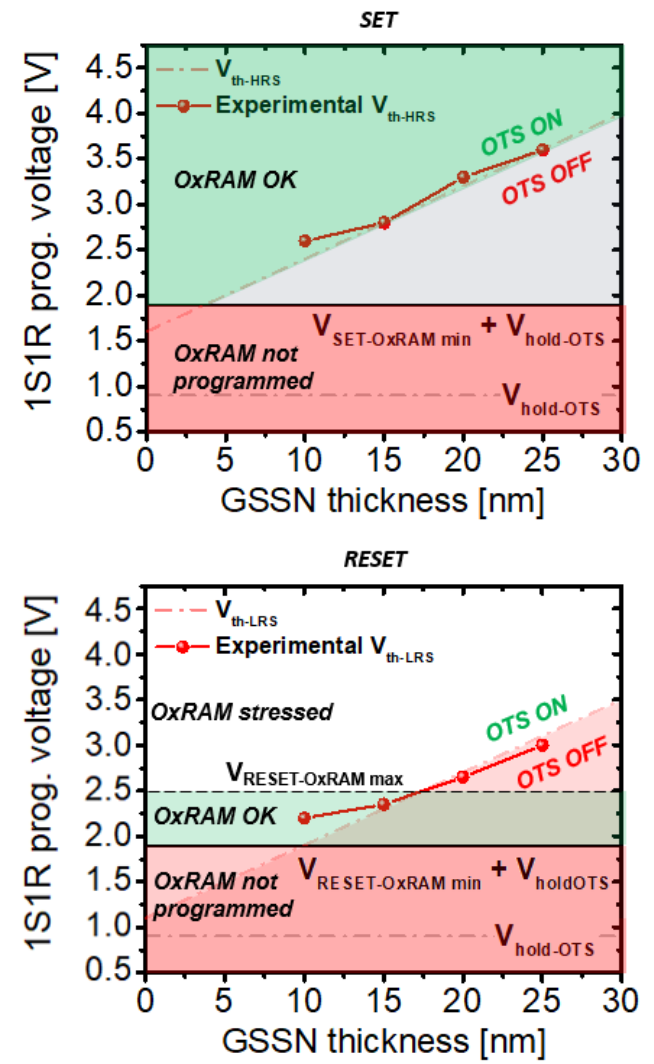

Fig. 13. 1S1R simulated and experimental switching voltage $\left(\mathrm{V}_{\text {th-HRS }}\right.$ and $\mathrm{V}_{\text {th- }}$ LRS) during SET and RESET. Functional operating regions are highligted where sufficient voltage is provided to both open the OTS and program the OxRAM.

Then, the impact of programming conditions on reading margin is evaluated for $15 \mathrm{~nm}$ GSSN (Fig.14-15). Functional behavior with a stable read margin is achieved for adapted SET and RESET conditions, allowing to reach $10^{7}$ cycles for optimized SET and RESET conditions and optimized GSSN thickness.

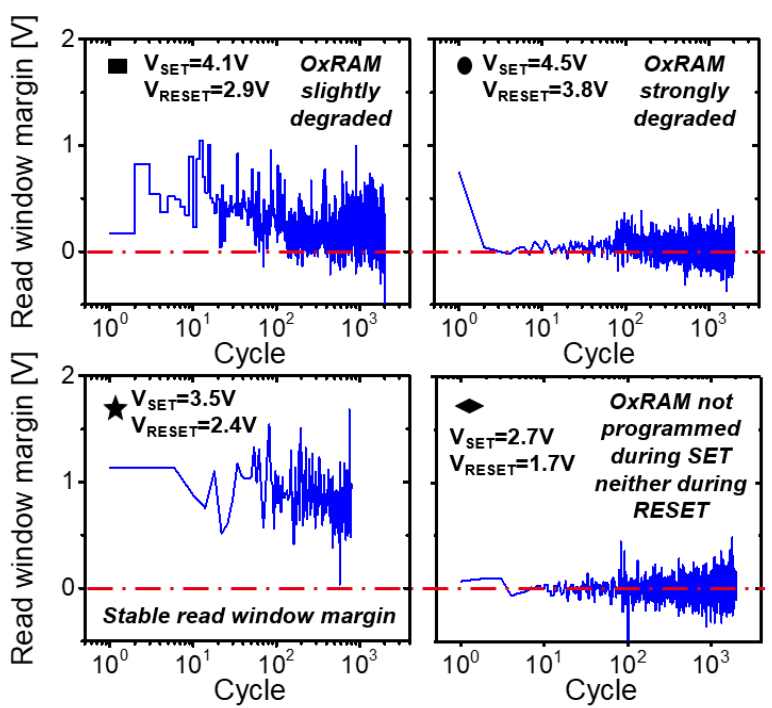

Fig. 14. Evolution of 1S1R read voltage margin over cycling for various programming conditions.



Fig. 15. 1S1R endurance characteristics for adapted programming condition (star condition Fig. 13). Fails are indicated in red.

Fig.16 generalizes the trend and links the programming voltages with $1 \mathrm{~S} 1 \mathrm{R}$ functionality as a function of OTS $\mathrm{V}_{\text {th }}$, based on the minimum and maximum voltages the OxRAM can sustain. Experimental cycling results (from Fig.14) are indicated on the graph, confirming the calculated optimized SET and RESET voltages. OTS $\mathrm{V}_{\text {th }}$ and leakage current being linked [4], the maximum bank size of the 1S1R can also be estimated, as reported on top of the graph. 

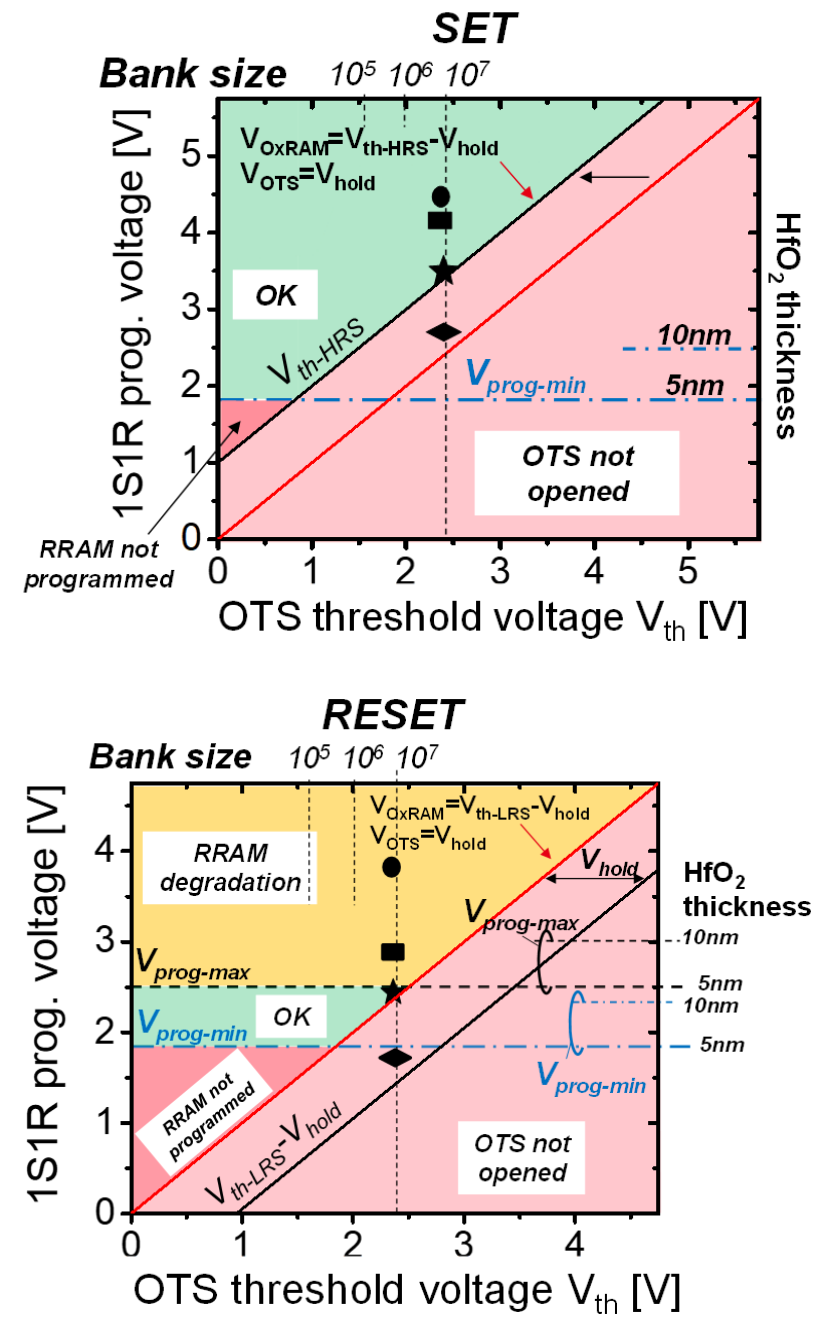

Fig. 16. Functional 1S1R SET and RESET voltages operating regions as function of OTS $V_{\text {th }}$. Functionality ensures OTS opening and sufficient voltage to program the OxRAM preventing device degradation. Symbols correspond to illustrative experimental cases showed in Fig.13.

Finally, Fig.17 summarizes $\mathrm{HfO}_{2}$ and GSSN thicknesses dependence to insure a reliable operating region and good overall 1S1R characteristics, including OxRAM integrity, large bank size and acceptable firing voltage.

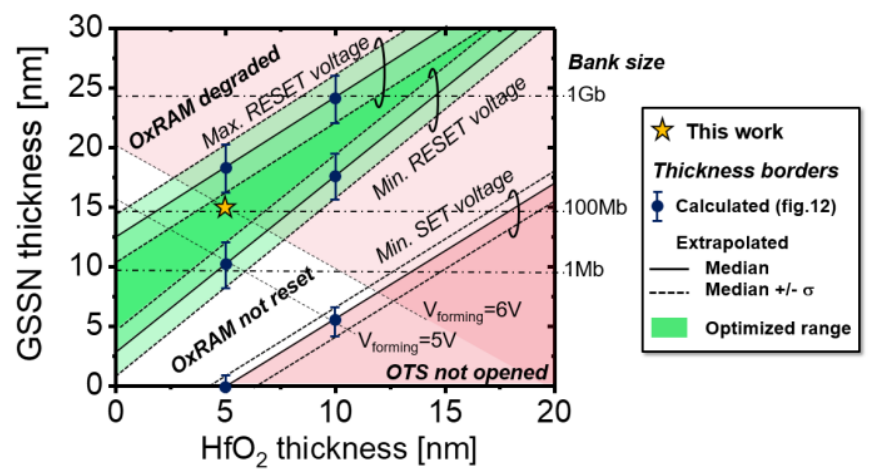

Fig. 17. $1 \mathrm{~S} 1 \mathrm{R}$ operating regions matching the $1 \mathrm{~S}$ and $1 \mathrm{R}$ stacks, playing with GSSN and $\mathrm{HfO}_{2}$ thicknesses. Good functionnality is insured when opening the OTS allows to program the OxRAM without degradation. $\mathrm{V}_{\text {th-LRS }}, \mathrm{V}_{\text {hold }}$ (simulated, see section $\mathrm{B}$ ), $\mathrm{V}_{\text {th-HRS }}$ (calculated, see section $\mathrm{C}$ a)) are used to simulate the operating limits.

\section{CONCLUSIONS}

1S1R switching operations are clarified by crossing numerical model and semi-analytical simulated results with experimental data. 1S1R dynamical voltage repartition and device degradation are elucidated. We provided general guidelines allowing to design $1 \mathrm{~S} 1 \mathrm{R}$ stack for optimized read window margin, large maximum array size and extended endurance. It appears that $10^{7}$ programming cycles can be achieved for adjusted SET and RESET conditions and optimized thicknesses of GSSN OTS and $\mathrm{HfO}_{2}$ OxRAM.

\section{Acknowledgments:}

This work was partially funded by European commission, French State and Auvergne-Rhône Alpes region through ECSEL project WAKEMEUP and French Nano2022 program.

\section{References:}

[1] S. Yasuda et al., "A Cross Point Cu-ReRAM with a Novel OTS Selector for Storage Class Memory Applications," in VLSI Tech. Dig. 2017, T30-31.

[2] K. Ohba et al., "Cross Point Cu-ReRAM with BC-Doped Selector", in Proc. 2018 IEEE IMW

[3] G. Molas et al., "Crosspoint Memory Arrays: Principle, Strengths and Challenges", in Proc. of IMW 2020, pp.1-4.

[4] D. Alfaro Robayo et al., IEDM 2019 Tech. Dig., pp. 35.3.1-35.3.4.

[5] J. Minguet Lopez et al., Optimization of RRAM and OTS selector for advanced Low voltage CMOS Compatibility", in Proc. of IMW 2020, pp.1-4.

[6] A. Verdy et al., "Optimized Reading Window for Crossbar Arrays Thanks to Ge-Se-Sb-N-based OTS Selectors", in IEDM 2018 Tech. Dig., 37.4.

[7] J. Sandrini et al., "OxRAM for embedded solutions on advanced node: scaling perspectives considering statistical reliability and design constraints", in IEDM 2019 Tech. Dig., pp. 30.5.1-30.5.4.

[8] V. G. Karpov et al., "Field-reduced nucleation in phase change memory", in Phys. Rev. B 78, 052201 (2008). 